\title{
Sherlock: An Automated Follow-Up Telescope for Wide-Field Transit Searches
}

\author{
Lewis Kotredes, David Charbonneau, Dagny L. Looper \\ and Francis T. O’Donovan \\ California Institute of Technology, M/C 105-24, 1200 E California Blvd, Pasadena, CA 91125; \\ ltk,dc,ftod@astro.caltech.edu,dagny@caltech.edu
}

\begin{abstract}
The most significant challenge currently facing photometric surveys for transiting gasgiant planets is that of confusion with eclipsing binary systems that mimic the photometric signature. A simple way to reject most forms of these false positives is high-precision, rapid-cadence monitoring of the suspected transit at higher angular resolution and in several filters. We are currently building a system that will perform higher-angular-resolution, multi-color follow-up observations of candidate systems identified by Sleuth (our wide-field transit survey instrument at Palomar), and its two twin system instruments in Tenerife and northern Arizona.
\end{abstract}

\section{INTRODUCTION}

Wide-field photometric surveys for transits of short-period gas-giant planets consist of several months of single-band observations of typically 5000 targets in a six-degreesquare field of view. A number of such surveys, including the network consisting of Sleuth ${ }^{1}$ (Palomar, PI: D. Charbonneau, see [1]), STARE ${ }^{2}$ (Tenerife, PI: T. Brown) and PSST (Lowell, PI: E. Dunham) have produced several candidates with light curves very similar to that of HD 209458 [2]. The most significant challenge facing such surveys is not the difficulty of obtaining the requisite precision and phase coverage, but rather the ability to rule out the large number of false positives that are typically encountered. These false positives can be removed through high-resolution spectroscopy, as was done in the case of the OGLE-III transit candidates [3, 4]. However, for relatively bright stars, there is an easier way to reject such candidates.

Brown [5] discusses various transit-like signals typically identified by searches similar to Sleuth and classifies them into a number of specific types. There are three primary sources of these false positives. The first of these are binary stars undergoing a grazing eclipse (MSU). The second and third types involve blends between a binary star undergoing a deep eclipse and a third star, either a foreground object (MSDF) or a third member of the system (MSDT). Using a typical transit campaign for the STARE instrument, Brown calculates that for every 10000 stars observed, 0.39 planets will be detected with three transits. However, 2.27 false positives of type MSU will also appear, 1.26 false

1 http://www.astro. caltech.edu/ ftod/sleuth.html

2 http://www.hao.ucar. edu/public/research/stare/stare.html 


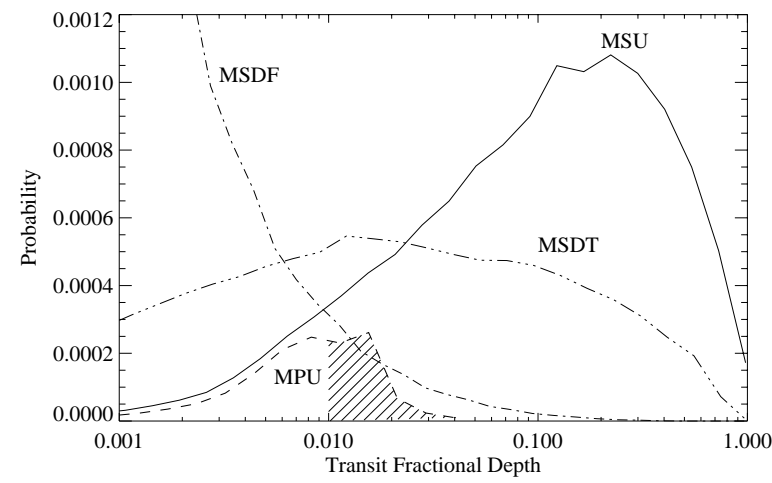

FIGURE 1. Taken from [5], the marginalized probabilities of detection of a transit per unit log transit depth. The shaded area is represents planets detectable in current surveys. Figure labels are explained in the text.

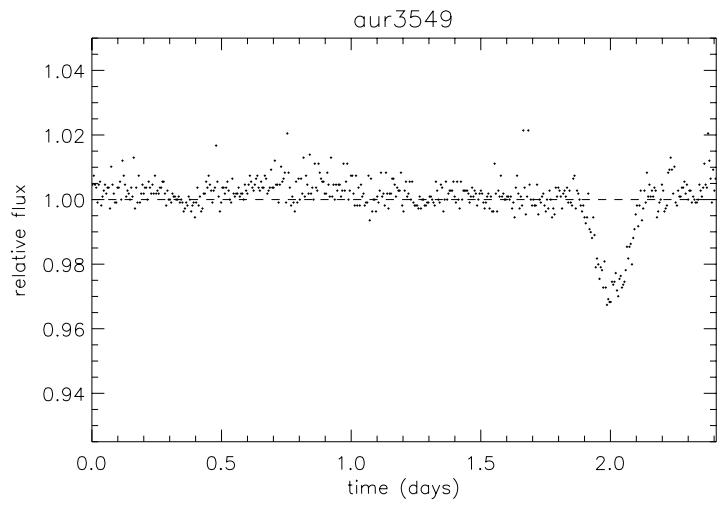

FIGURE 2. Phased R-band light curve of a star in Auriga as observed with the PSST by E. Dunham \& G. Mandushev (Lowell Observatory). Based on this light curve alone, this appears to be a reasonable candidate, as the period, duration, and depth are consistent with the passage of an inflated gas-giant across a Sun-like star. However, follow-up photometry shows this object to be a blend containing an eclipsing binary (see Figure 3).

positives of type MSDF will be present, and 0.98 candidates of type MSDT will be observed. Combining these numbers, Brown finds that a transit survey will observe more than 10 false positives for every true planet. This is demonstrated by Figure 1, which shows the probability of finding different classes of object at a given transit depth. In the regime of 1-2\% eclipse depths that we study, these three sources of false positives are at least as plentiful as the planets we seek. 


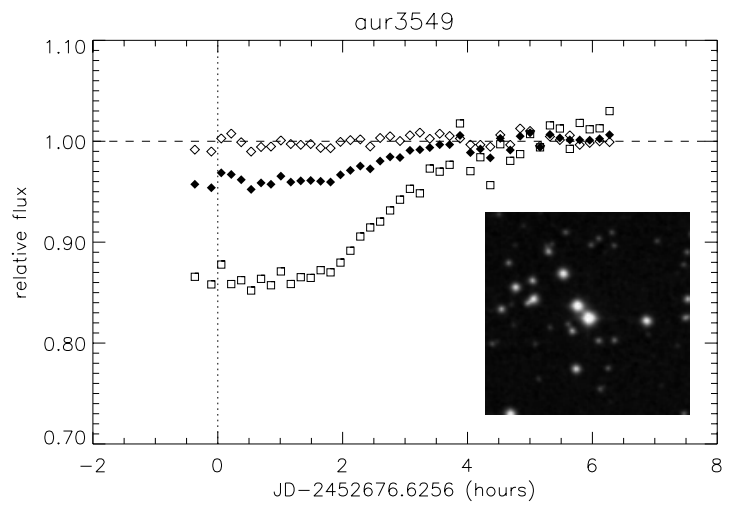

FIGURE 3. The inset Digitized Sky Survey image shows this target to consist of a central bright source and an adjacent fainter star to the NE. Both of these stars are contained within the PSF of the PSST. We carried out follow-up photometry of this field with a 14-inch telescope located on the roof of the Caltech Astronomy building, during a night when an eclipse was predicted to occur. Observations began during mid-eclipse. The predicted time of eclipse occurs at $t=0$ of this figure. The open diamonds show the light curve for the central star, which is evidently uneclipsed. The open squares show the light curve for the star to the NE, which undergoes a deep (14\%) eclipse. When the light from both stars is summed in a single aperture, the apparent eclipse depth is reduced to $\sim 3 \%$, reproducing the PSST light curve (see Figure 2).

\section{SHERLOCK: REMOVING THE ECLIPSING BINARIES}

We are currently assembling a telescope named Sherlock that will be dedicated to examining transit candidates from Sleuth, as well as a number of other transit surveys which monitor stars brighter than $\mathrm{V}=13$. Highly automated and observing in custom RGB filters with a better angular resolution (1.7 $\mathrm{arcsec} / \mathrm{pixel})$ than Sleuth (10 arcsec/pixel), Sherlock will be able to reject most of the contaminants from these transit surveys. In so doing, Sherlock will greatly reduce the rate of false positives, bringing the number of transit candidates to a manageable level. Figures $2 \& 3$ show an example of how blends of eclipsing binaries can be separated from transiting planets. PSST identified a star in Auriga undergoing $2.5 \%$ deep eclipses with a period of 2.4 days (Figure 2). Examination of the Digitized Sky Survey images (Figure 3) revealed two sources within the PSF of the PSST instrument. Photometry of the field during a subsequent eclipse with a 14-inch telescope on the rooftop of the Caltech Astronomy building demonstrated that the fainter star was undergoing deep (14\%) eclipses. When the light from both stars was summed within a single photometric aperture, the transit shape and depth as observed by the PSST was reproduced (Figure 3).

Measurement of the color dependence of the transit depth should remove both grazing incidence binaries (due to the effect of limb-darkening on the eclipse depth), and blends of eclipsing binaries (due the change in relative brightness of the blended and occulted stars as a function of color). In contrast to this, planetary transits should be nearly constant with color. Furthermore, the increased angular resolution will separate the light from physically unassociated blended stars, and subsequent photometry will reveal 
TABLE 1. Specifications of Sherlock

Meade LX200GPS 10 ” f/6.3 Schmidt-Cassegrain Telescope
Apogee 1024 x 1024 pixel back-illuminated CCD camera
Filter wheel containing custom RGB and clear filters
SBIG STV Autoguider
Automated operation controlled by Linux workstation
Cloud cover monitored by Snoop, the Palomar All-Sky Camera

which object is undergoing eclipses. Sherlock will not be able to reject all sources of false detection. Central eclipses by very dim stellar objects (notably $\mathbf{M}$ dwarfs) will not show detectable variation with color. In addition, blends wherein the occulted and blending star have the same color will not be distinguished. Even with these exceptions, however, Sherlock is expected to reduce greatly the ratio of false positives from these surveys. Multi-epoch spectra of viable candidates will be gathered with high-resolution spectrographs on 1-2 m class telescopes, which should rule out the presence of stellar or brown dwarf companions. Surviving candidates will be monitored with Keck HIRES to determine the radial velocity orbit induced by the planetary companion.

\section{SHERLOCK SPECIFICATIONS}

Table 1 lists the specifications for Sherlock. The system will be located in the same clamshell enclosure as Sleuth, our primary transit search instrument. Weather decisions are made by the on-site 200-inch telescope night assistant, with additional protection provided by a weather station capable of closing the clamshell roof. Sherlock will be completely automated, calculating future times of eclipse for all active candidates, and observing the highest priority object in eclipse each night. The automated nature of the system is an advantage over comparatively labor- and resource-intensive multi-epoch spectroscopic follow-up. In addition, this dome hosts the new all-sky camera Snoop ${ }^{3}$, which provides weather monitoring for the observatory. Given that observing time on this system should be plentiful, we invite teams conducting wide-field transit surveys to contact us regarding follow-up of their candidates.

\section{REFERENCES}

1. O'Donovan, F. T., Charbonneau, D., and Kotredes, L., 2003, AIP Conf Proc: The Search for Other Worlds, eds. S. S. Holt \& D. Deming, (Springer-Verlag), astro-ph/0312289

2. Charbonneau, D., Brown, T. M., Latham, D. W., and Mayor, M., 2000, ApJ, 529, 245

3. Konacki, M., Torres, G., Jha, S., and Sasselov, D. D., 2003, Nature, 421, 507

4. Konacki, M., Torres, G., Sasselov, D. D., and Jha, S., 2003, ApJ, 597, 1076

5. Brown, T., 2003, ApJ, 593, L125

${ }^{3}$ http: //snoop.palomar.caltech.edu 